\title{
PERFIL ANTROPOMÉTRICO E PARÂMETROS CARDIOVASCULARES DOS PARTICIPANTES DE UM PROGRAMA DE PROMOÇÃO DA SAÚDE DE ARARANGUÁ/SC
}

\author{
Viviane José de Oliveira Bristot \\ Universidade Federal de Santa Catarina \\ vivianijo@hotmail.com \\ Danielle Soares Rocha Vieira \\ Universidade Federal de Santa Catarina \\ danielle.vieira@ufsc.br
}

\author{
Ione Jayce Ceola Schneider \\ Universidade Federal de Santa Catarina \\ ione.schneider@ufsc.br \\ Viviane de Menezes Caceres \\ Universidade Federal de Santa Catarina \\ viviane.caceres@ufsc.br
}

Resumo

Este estudo buscou investigar os parâmetros antropométricos e cardiovasculares de participantes de um programa de extensão em promoção da saúde na cidade de Araranguá (Santa Catarina, Brasil), bem como seus níveis de atividade física. Foi realizado um estudo transversal no período de julho a dezembro de 2015. A coleta dos dados foi realizada com 323 indivíduos com idade entre 18 a 90 anos, e observou-se alta prevalência de sobrepeso e obesidade (70,5\%) e hipertensão autorreferida (43,3\%). Os fatores de risco encontrados convergem para os mais prevalentes na população, excesso de peso e hipertensão. Este levantamento auxilia na definição de novos programas de promoção e prevenção além de fortalecer as estratégias de saúde do município.

Palavras-chave: Promoção da Saúde. Prevenção de Doenças. Fatores de Risco Cardiovascular.

\section{ANTHROPOMETRIC PROFILE AND CARDIOVASCULAR PARAMETERS OF PARTICIPANTS OF A PROGRAM OF PROMOTION OF HEALTH OF ARARANGUÁ/SC}

Abstract

This study aimed to investigate the anthropometric and cardiovascular profile of participants in a health promotion program in the city of Araranguá (Santa Catarina, Brazil), as well as, their levels of physical activity. A cross-sectional study was carried out from July to December of 2015. Data collection was performed within 323 individuals aged between 18 and 90 years, and a high prevalence of overweight and obesity $(70.5 \%)$ and Hypertension (43.3\%) was observed. The risk factors converge to the most prevalent in the population, being overweight and hypertensive. This survey helps in the definition of new promotion and prevention programs, besides strengthening the health strategies of the municipality.

Keywords: Health Promotion. Disease Prevention. Cardiovascular Risk Factors.

\section{PERFIL ANTROPOMÉTRICO Y PARÁMETROS CARDIOVASCULAR DE PARTICIPANTES DE UN PROGRAMA DE PROMOCIÓN DE LA SALUD DE ARARANGUÁ/SC}

\section{Resumen}

Este estudio tuvo como objetivo investigar los parámetros antropométricos y cardiovasculares de los participantes en un programa de promoción de la salud en la ciudad de Araranguá, así como sus niveles de actividad física. Un estudio de la sección transversal en el periodo de julio a diciembre de 2015. La recogida de datos se realizó con 323 sujetos de edad 18 90 años y se observó una alta prevalencia del sobrepeso y la obesidad $(70,5 \%)$ y dicho hipertensión auto (43,3\%). Los factores de riesgo encontraron a converger el más prevalentes en la población, el sobrepeso y la hipertensión. Esta encuesta ayuda en la definición de nuevos programas de promoción y prevención, así como el fortalecimiento de las estrategias de salud del municipio.

Palavras clave: Promoción de la Salud. Prevención de Enfermedades. Los factores de riesgo cardiovascular. 
Perfil antropométrico e parâmetros cardiovasculares dos participantes de um programa de promoção da saúde de Araranguá/SC

\section{INTRODUÇÃO}

Promoção da saúde e prevenção de doenças são abordagens para atender as demandas atuais e futuras com a saúde, através de ações integradas e intersetoriais (Long et al., 2014; Wang et al., 2014; Knapper et al., 2015). Promover comportamentos de saúde auxiliam na manutenção de níveis recomendados de atividade física, massa corporal e dieta saudável (Tucker et al., 2014; Thomson et al., 2015; Vedanthan et al., 2016). O Plano de Ações Estratégicas para o Enfrentamento das Doenças Crônicas Não Transmissíveis no Brasil, abrange os grupos de agravos: cardiovasculares; cânceres; diabetes; e doenças respiratórias crônicas (Brasil, 2011).

Dentro desse contexto, programas de promoção da saúde bem formulados tem se tornado um meio promissor na redução de alguns fatores de risco para doenças cardiovasculares (DCV) como, as alterações no índice de massa corporal (IMC), glicemia, pressão arterial e colesterol total (Wu et al., 2012; Campbell et al., 2014; Eng et al., 2016).

O IMC, em geral, correlaciona-se bem com outras medidas da gordura corporal, e é amplamente empregado em estudos da obesidade (Barreira et al., 2012; Sarma et al., 2014). Segundo alguns autores, os fatores comportamentais e culturais interferem diretamente para um IMC anormalmente elevado, entre os quais estão a ingestão de calorias em excesso e sedentarismo (Burrows et al., 2016). Esta combinação promove alterações morfológicas e fisiológicas que resultam em distúrbios metabólicos crônicos como o diabetes (Hesselink et al., 2013).

A prevalência de distúrbios metabólicos está crescendo rapidamente, e consequentemente, aumentando os custos médicos e diminuindo a qualidade de vida para os pacientes e suas famílias (Zhang and Padman, 2016). A hiperglicemia provocada pelo diabetes juntamente com a dislipidemia contribuem significativamente para a maior incidência de DCV (Vázquez et al., 2014).

O treinamento físico tem demonstrado moderada redução na pressão arterial sistólica (PAS) e diastólica (PAD) em cerca de $10 \mathrm{mmHg}$, ou seja, atividade física tem importante papel preventivo na DCV (Pattyn et al., 2013; García-Ortiz et al., 2014; Bouaziz et al., 2015; Skrypnik et al., 2015; Venturelli et al., 2015). Além disso, muitos estudos sugerem que a inatividade física é reconhecida como importante fator de risco para múltiplas causas de morbimortalidade de doenças crônicas (Belardinelli et al., 2012; Naci and Ioannidis, 2013; Loprinzi and Davis, 2015; Brawner et al., 2016).

Um dos instrumentos utilizados para medir o nível de atividade física é o Questionário 
Perfil antropométrico e parâmetros cardiovasculares dos participantes de um programa de promoção da saúde de Araranguá/SC

Internacional de Atividade Física (IPAQ) que avalia o volume e intensidade da atividade física habitual e comportamento sedentário de maneira simples e eficaz para atender não somente restrições espaciais, mas também temporais (Lee et al., 2012; Jerônimo et al., 2014; Nakamura et al., 2014; Andersson et al., 2015; Nunes et al., 2015; Rasiah et al., 2015). Estudos recentes verificaram que os dados recolhidos a partir de questionários de atividade física são usados para examinar associações entre atividade física e saúde, e para permitir associações de interesse na pesquisa epidemiológica (Daniele et al., 2013; Minder et al., 2014).

Os programas de extensão e educação em saúde na comunidade contribuem na construção de ações que possibilitam responder às necessidades sociais em saúde (WHO, 2016). O programa de promoção de saúde, Sesc Saúde consiste em um programa de ações com ênfase na prevenção de fatores determinantes e condicionantes de doenças e agravos à saúde, incidindo sobre as condições de vida e favorecendo a ampliação de escolhas saudáveis por parte dos sujeitos no território onde vivem e trabalham. Diante do exposto, este estudo teve como objetivo investigar de forma transversal os parâmetros antropométricos, cardiovasculares e nível de atividade física de participantes de um programa de extensão em promoção da saúde na cidade de Araranguá- SC.

\section{MATERIAIS E MÉTODOS}

Trata-se de um estudo transversal, de caráter exploratório e descritivo, em que foram avaliados participantes de uma programação mensal de atividades voltadas a nove diferentes temas sobre saúde, no período de julho a dezembro de 2015, no município de Araranguá. O estudo foi aprovado sob o Certificado de Apresentação para Apreciação Ética número (CAAE) 55524116.6.0000.0121.

A amostra desse estudo foi composta por participantes de um programa de extensão em promoção de saúde da cidade de Araranguá-SC, chamado Sesc Saúde. Todos os interessados a responder o questionário foram incluídos se tivessem idade igual ou superior a 18 anos. Foram excluídos indivíduos com incapacidade de compreender ou realizar os procedimentos do protocolo de pesquisa. Como o objetivo foi avaliar o perfil dos participantes de um programa específico de promoção à saúde que ocorria mensalmente na cidade de Araranguá, a amostragem foi feita por conveniência no período de 6 meses.

A coleta de dados foi realizada no local selecionado pela entidade parceira para desenvolver o programa de promoção da saúde. Foi providenciado junto ao setor de Comunicação desta Instituição a divulgação do programa. Os pesquisadores juntamente com funcionários da instituição parceira estabeleceram uma programação prévia das atividades a serem 
Perfil antropométrico e parâmetros cardiovasculares dos participantes de um programa de promoção da saúde de Araranguá/SC

realizadas conforme a temática do mês. No dia pré-determinado para desenvolvimento da atividade, foram realizadas orientações de saúde sobre a temática do mês, o qual fez parte os temas ou assuntos "Saúde Ocular, Doenças Crônicas não Transmissíveis (DCNT), Saúde do Idoso, Câncer de Mama, Câncer de Próstata, HIV, AIDS, e Hepatite”, além das coletas dos dados demográficos, antropométricos e glicêmicos. Os participantes também responderam um questionário sobre nível de atividade física e questionados sobre a presença de doenças respiratórias, cardiovasculares e seus fatores de risco.

A avaliação antropométrica foi determinada pela massa corporal, obtida utilizando uma balança digital marca Sanny®, com capacidade para $150 \mathrm{~kg}$ e escala de $100 \mathrm{~g}$, aferida e posicionada em superfície plana; e para mensuração da estatura, estadiômetro compacto, marca Sanny®, com capacidade de $210 \mathrm{~cm}$, resolução em $\mathrm{mm}$, firmemente fixado a uma superfície vertical plana (parede sem rodapé). As aferições, tanto da massa corporal quanto da estatura foram realizadas uma única vez e seguiram as recomendações World Health Organization (WHO, 2015).

A pressão arterial foi mensurada no membro superior direito utilizando-se esfigmomanômetro calibrado (Tycos, Louisiana, USA) e estetoscópio (Littmann Classic II, São Paulo, Brasil), seguindo as recomendações da Sociedade Brasileira de Cardiologia (2010). Após, foi mensurada a frequência cardíaca no pulso radial, cronometrado por 60 segundos.

O IMC (peso/altura ${ }^{2}$ ) foi utilizado para classificar os participantes como apresentando baixo peso, peso normal, sobrepeso ou obesidade de acordo com os critérios da Organização Mundial de Saúde (WHO, 2015).

A versão curta do IPAQ foi utilizada para medida do nível de atividade física, que é composta por oito questões abertas e suas informações permitem estimar o tempo despendido em diferentes dimensões de atividade física. Os usuários foram classificados em muito ativo, ativo, irregularmente ativo A, irregularmente ativo B ou sedentário. Os indivíduos classificados como muito ativo apresentaram um dos dois critérios seguintes: 1) 3 ou mais dias de atividade vigorosa por pelo menos 20 minutos por dia, mais 5 ou mais dias de atividade de intensidade moderada ou caminhada por pelo menos 30 minutos por dia; ou 2) 5 ou mais dias de atividade vigorosa e 30 ou mais minutos por sessão. Os indivíduos classificados como ativos realizaram um dos três critérios seguintes: 1) qualquer combinação de caminhada, de intensidade moderada ou atividades de intensidade vigorosa, que somadas atingiram 5 ou mais dias na semana e 30 ou mais minutos por sessão; ou 2) atividade moderada ou caminhada, 5 ou mais dias na semana e 30 ou mais minutos por sessão; ou 3) atividade vigorosa, 3 ou mais dias na semana e 20 ou mais minutos por sessão. Aqueles indivíduos que não satisfazem os critérios para as categorias 1 ou 2 
Perfil antropométrico e parâmetros cardiovasculares dos participantes de um programa de promoção da saúde de Araranguá/SC

são considerados irregularmente ativos. Os indivíduos considerados irregularmente ativos foram divididos em dois subgrupos de acordo com a soma da frequência e duração dos diferentes tipos de atividades. São eles: os irregularmente ativo A, sendo aqueles que cumpriram a frequência de 5 dias na semana ou duração de 150 minutos da semana, e os irregularmente ativos B, aqueles que não atingiram nenhum dos critérios da recomendação quanto à frequência nem quanto á duração. Para os indivíduos classificados como sedentários, foram considerados aqueles que não realizavam nenhuma atividade física por pelo menos 10 minutos contínuos durante a semana. (Guidelines for Data Processing and Analysis of the International Physical Activity Questionnaire -IPAQ, 2004).

Os dados foram analisados através de medidas de tendência central e dispersão, para variáveis quantitativas, e frequência relativa e absoluta, para as qualitativas, geral e estratificadas por sexo. Para testar associação foi realizada teste qui-quadrado para variáveis categóricas, e testes de média para as variáveis contínuas. As análises estatísticas foram realizadas por meio do pacote estatístico SPSS versão 17.0. Foram excluídos aqueles com dados ausentes ou discrepantes.

\section{RESULTADOS E DISCUSSÃO}

No período de atividades do programa de promoção da saúde houve a participação de 323 indivíduos com idades entre 18 a 90 anos e média de 55 anos, e as mulheres representaram 61\% dos participantes. A Tabela 1 mostra as médias e os desvios padrões das características glicêmicas, antropométricas e hemodinâmicas, geral e estratificadas por sexo. Em relação a estratificação por sexo, as mulheres apresentaram menores valores de massa corporal e menor estatura, o que levou a maiores valores de IMC. Também apresentaram menores valores de PAS e PAD (Tabela 1).

Tabela 1: Descrição das características demográficas, antropométricas, parâmetros hemodinâmicos, e sanguínea, estratificados por sexo.

\begin{tabular}{lrrrrrr}
\hline \multirow{2}{*}{ Características } & \multicolumn{2}{c}{ Geral } & \multicolumn{2}{c}{ Homens } & \multicolumn{2}{c}{ Mulheres } \\
\cline { 2 - 7 } & $\mathrm{n}$ & \multicolumn{1}{c}{ Média (DP) } & \multicolumn{1}{c}{$\mathrm{n}$} & Média (DP) & \multicolumn{1}{c}{$\mathrm{n}$} & Média (DP) \\
\hline Idade (anos) & 323 & $55,4(14,7)$ & 126 & $55,3(15,5)$ & 197 & $55,5(14,2)$ \\
Peso (kg) & 321 & $76,2(14,8)$ & 124 & $81,8(14,9)$ & 197 & $72,7(13,7)$ \\
Altura (cm) & 323 & $1,4(0,4)$ & 112 & $1,51(0,5)$ & 182 & $1,4(0,4)$ \\
Índice de massa corporal $\left(\mathrm{kg} / \mathrm{m}^{2}\right)$ & 323 & $26,0(9,8)$ & 110 & $25,0(10,6)$ & 182 & $26,7(0,4)$ \\
Pressão arterial sistólica $(\mathrm{mmHg})$ & 306 & $135,2(19,7)$ & 119 & $140,0(19,8)$ & 187 & $132,1(19,1)$ \\
Pressão arterial diastólica $(\mathrm{mmHg})$ & 305 & $76,4(11,5)$ & 119 & $77,7(13,4)$ & 187 & $75,5(10,0)$ \\
Frequência cardíaca (bpm) & 292 & $76,1(12,1)$ & 114 & $74,9(13,1)$ & 178 & $76,9(11,5)$ \\
\hline
\end{tabular}


Perfil antropométrico e parâmetros cardiovasculares dos participantes de um programa de promoção da saúde de Araranguá/SC

O IMC foi categorizado e apresentou a seguinte distribuição: abaixo do peso: $0,0 \%$; peso normal: 29,4\%; sobrepeso: 36,5\%; e obesidade: 34,0\%. Ao estratificar por sexo, não existiram homens e mulheres classificados abaixo do peso, 31,7\% apresentou peso normal, 36,5\% e 31,7\% com sobrepeso e obesidade, respectivamente. Entre as mulheres, 27,9\% peso normal, 36,5\% sobrepeso e $35,5 \%$ obesidade.

Não foi observada associação entre IMC com outras variáveis hemodinâmicas ou a presença de doenças cardiovasculares, assim como pressão arterial e as variáveis investigadas. Diferente de nossos resultados, em um estudo realizado por Roever e colaboradores em Uberlândia (MG, Brasil), foi observado uma correlação positiva dos parâmetros relacionados com a obesidade com fatores de risco metabólicos, sendo que tal correlação apresentou uma diferença entre os gêneros (Roever et al., 2016).

Já Suemoto et al. em um estudo de coorte realizado com idosos em São Paulo, observou que $68 \%$ da população apresentou sobrepeso e obesidade. Neste estudo, a prevalência de obesidade associada a diabetes aumentou o risco de morte(Suemoto et al., 2015).

Sabe-se que excesso de peso é fator de risco de muitas doenças do sistema cardiovascular (American College of Cardiology/American Heart Association Task Force on Practice Guidelines, 2014). Dentre as diversas substâncias liberadas pelo tecido adiposo, estão as adipocinas. O excesso de peso favorece o aumento da liberação de adipocinas na corrente sanguínea, que além de estimular uma maior expansão do tecido adiposo também podem induzir um perfil inflamatório. Assim, este seria um dos mecanismos pelos quais este fator de risco favorece o desenvolvimento e progressão das doenças cardiovasculares no contexto da obesidade e diabetes do tipo 2 (Northcott et al., 2012).

Em relação a pressão arterial, nosso estudo demonstrou que a média da pressão arterial sistólica dos homens foi de $140 \mathrm{mmHg}$. Esse valor encontra-se classificado como hipertensos estágio 1, já a média da pressão arterial sistólica das mulheres foi de $132 \mathrm{mmHg}$, que se encontra classificado como pressão limítrofe segundo a VI Diretrizes Brasileiras de Hipertensão (SOCIEDADE BRASILEIRA DE CARDIOLOGIA, 2010). A pressão arterial diastólica apresentou valores de $77 \mathrm{mmHg}$ e $75 \mathrm{mmHg}$ para homens e mulheres, respectivamente. 
Perfil antropométrico e parâmetros cardiovasculares dos participantes de um programa de promoção da saúde de Araranguá/SC

Tabela 2: Descrição das variáveis de saúde, estratificadas por sexo.

\begin{tabular}{|c|c|c|c|}
\hline Variáveis & \multicolumn{2}{|c|}{ n (\%) } & Valor de $\mathrm{p}$ \\
\hline Categoria de IMC & Homens $(n=126)$ & Mulheres $(n=197)$ & \\
\hline Abaixo do peso & $0(0)$ & $0(0)$ & 0,702 \\
\hline Peso normal & $40(31,7)$ & $55(27,9)$ & \\
\hline Sobrepeso & $46(36,5)$ & $72(36,5)$ & \\
\hline Obesidade & $40(31,7)$ & $70(35,5)$ & \\
\hline Atividade física & Homens $(n=126)$ & Mulheres $(n=197)$ & \\
\hline Ativo & $69(54,8)$ & $121(61,4)$ & 0,202 \\
\hline Irregularmente ativo A e B & $29(23,0)$ & $42(21,3)$ & \\
\hline Muito ativo & $13(10,3)$ & $23(11,7)$ & \\
\hline Sedentário & $15(11,9)$ & $11(5,6)$ & \\
\hline Diagnóstico de diabetes & Homens $(n=126)$ & Mulheres $(n=197)$ & \\
\hline Não & $94(74,6)$ & $160(81,2)$ & 0,157 \\
\hline $\operatorname{Sim}$ & $32(25,4)$ & $37(18,8)$ & \\
\hline Diagnóstico de hipertensão & Homens $(n=126)$ & Mulheres $(n=197)$ & \\
\hline Não & $80(63,5)$ & $103(52,3)$ & 0,047 \\
\hline $\operatorname{Sim}$ & $46(36,5)$ & $94(47,7)$ & \\
\hline $\begin{array}{l}\text { Diagnóstico de colesterol ou } \\
\text { triglicérides elevados }\end{array}$ & Homens $(n=126)$ & Mulheres $(n=197)$ & \\
\hline Não & $89(70,6)$ & $137(69,5)$ & 0,835 \\
\hline $\operatorname{Sim}$ & $37(29,4)$ & $60(30,5)$ & \\
\hline Histórico de doença crônica & & & \\
\hline Doença Respiratória & Homens $(n=126)$ & Mulheres $(n=197)$ & \\
\hline Não & $112(88,9)$ & $170(86,3)$ & 0,494 \\
\hline $\operatorname{Sim}$ & $14(11,1)$ & $27(13,7)$ & \\
\hline Doença Cardíaca & Homens $(n=126)$ & Mulheres $(n=197)$ & \\
\hline Não & $103(81,7)$ & $156(79,2)$ & 0,574 \\
\hline Sim & $23(18,3)$ & $41(20,8)$ & \\
\hline
\end{tabular}

Adicionalmente, um estudo de coorte observou 1784 indivíduos do nascimento até 60-64 anos de idade e demonstrou associação entre obesidade e o aumento de fatores inflamatórios, 
Perfil antropométrico e parâmetros cardiovasculares dos participantes de um programa de promoção da saúde de Araranguá/SC

enfatizando que os resultados podem contribuir para a disfunção vascular e consequente aumento da pressão sanguínea devido a ligação com a via aterosclerótica (Murray et al., 2015). Outros estudos mostraram a tendência do aumento da pressão arterial com o aumento da idade, fato que pode ser devido ao desencadeamento das alterações fisiológicas próprias do processo de envelhecimento. (Ding et al., 2015; Lim et al., 2015; Maksuti et al., 2016).

A prevalência da hipertensão arterial sistêmica auto referida foi de 43,3\%. Chow et al em um estudo com 142.042 participantes de 17 países avaliou através de auto relato e medições a prevalência de hipertensão, conhecimento, tratamento e controle da doença. A prevalência de hipertensão auto referida foi de 46\%, sendo que desses apenas $25 \%$ realizam tratamento adequado com anti-hipertensivo (Chow et al., 2013).

Todas as atividades desenvolvidas mensalmente, principalmente as orientações segundo a temática de cada mês no programa de promoção da saúde, proporcionaram aos participantes aumentar o conhecimento sobre os fatores de risco de algumas doenças de maior prevalência no Brasil, o que gradativamente fez do programa um espaço produtivo, educativo e atraente, além de servir de palco para orientações feitas pelos alunos do projeto de extensão. Além das orientações os participantes do programa tiveram oportunidade de conhecer seus dados antropométricos e hemodinâmicos, o que estimulou a manter ou implementar bons hábitos de vida. Um estudo realizado no Brasil que incluiu 5779 adultos, considerou a importância das características ambientais como oportunidades ou barreiras na adoção de hábitos saudáveis como a prática de atividade física, aumentando a importância de estratégias de promoção da saúde (Gomes et al., 2016). Além disso, estudos recentes abordam as ações antiinflamatórias da atividade física como meio de modular mecanismos biológicos subjacentes ao processo de envelhecimento para retardar o aparecimento de doenças relacionadas à idade e promover um envelhecimento saudável (Chung et al., 2009).

Em relação as doenças respiratórias auto referidas, 12,6\% da amostra dos participantes do programa de promoção de saúde relataram apresentar algum tipo de doença respiratória. Dentre estes, 21,6\% relataram não saber o diagnóstico da doença. As doenças mais recorrentes foram bronquite crônica e asma brônquica com prevalência de 59,4\%.

Já a prevalência de doenças cardíacas auto referida foi de 19,8\%, que a proporção entre homens e mulheres foi de $18,3 \%$ e $20,8 \%$, respectivamente. A doença cardíaca mais relatada foi a arritmia cardíaca $(15,6 \%)$, e de todos que relataram apresentar algum tipo de doença cardíaca, 26,5\% não tinham conhecimento do diagnóstico da doença.

Neste cenário, programas de promoção de saúde se mostram importantes quanto ao papel da orientação para fazer as pessoas capazes de melhorar e aumentar o seu controle sobre 
Perfil antropométrico e parâmetros cardiovasculares dos participantes de um programa de promoção da saúde de Araranguá/SC

sua saúde. Os profissionais envolvidos podem identificar riscos para a saúde no estilo de vida do paciente, aconselhar e educar individualmente ou em grupos.

Com isso, o impacto do projeto na comunidade consistiu no aumento da confiança dos participantes em sua capacidade de lidar com a sua doença e a expectativa de melhorar fatores modificáveis de risco cardiovascular.

O presente estudo também contribui para o entendimento dos indivíduos a respeito de possíveis problemas de saúde e a importância da redução de fatores de risco cardiovascular como IMC e pressão arterial, bem como os benefícios de comportamentos positivos como a prática de exercício físico de forma regular e dieta saudável, uma vez que esta prática é recomendada para pacientes com hipertensão como uma opção de tratamento para reduzir os níveis de pressão em hipertensos e normotensos.

\section{CONSIDERAÇÕES FINAIS}

Este estudo encontrou que os participantes do programa de promoção de saúde analisado são majoritariamente mulheres, com média de idade de 55 anos, ativos, com excesso de peso, sem histórico de doenças crônicas, e hipertensão sistólica. Avaliar fatores de risco para doenças cardiovasculares em uma determinada amostra é um aspecto importante do processo de avaliação global da saúde para população em geral, em particular nos domínios de detecção precoce e prevenção de doenças crônicas.

As limitações deste estudo podem ter contribuído para que não houvessem relações entre as variáveis de interesse. Em primeiro lugar, um número reduzido da amostra provavelmente resultou em um poder estatístico limitado. Em segundo lugar, a auto seleção dos participantes que limita a capacidade de generalizar os achados do estudo para a população em geral. Estudos adicionais podem analisar maior amostra para possibilitar que os resultados sejam estendidos para todas as pessoas.

Um dos principais pontos fortes do estudo foi analisar separadamente homens e mulheres e descrever a prevalência de fatores de risco para DCV como, o sobrepeso e obesidade, hipertensão arterial sistêmica, colesterol ou triglicérides elevado e falta de atividade física, a fim de delinear ações a serem desenvolvidas como estratégias de promoção da saúde para os participantes.

Os resultados obtidos neste estudo, somado aos resultados de outros estudos que têm sido desenvolvidos e estão em andamento, fornecem informações sobre o estado de saúde de participantes do programa de extensão em promoção de saúde da cidade de Araranguá-SC, a fim 
Perfil antropométrico e parâmetros cardiovasculares dos participantes de um programa de promoção da saúde de Araranguá/SC

de auxiliar a implementação de serviços de saúde, envolvendo tanto ações específicas, como campanhas públicas do sistema de saúde que visam a prevenção dos principais fatores de risco, bem como orientações multidisciplinares com base científica sobre estudos realizados em populações semelhante.

\section{REFERÊNCIAS}

AMERICAN COLLEGE OF CARDIOLOGY/AMERICAN HEART ASSOCIATION TASK FORCE ON PRACTICE GUIDELINES, O. E. E. P., 2013. Executive summary: Guidelines (2013) for the management of overweight and obesity in adults: a report of the American College of Cardiology/American Heart Association Task Force on Practice Guidelines and the Obesity Society published by the Obesity Society and American College of Cardiology/American Heart Association Task Force on Practice Guidelines. Based on a systematic review from the The Obesity Expert Panel, 2013. Obesity (Silver Spring), v. 22 Suppl 2, p. S5-39, Jul 2014. ISSN 1930-739X. Available at: < https://www.ncbi.nlm.nih.gov/pubmed/24961825 >.

ANDERSSON, M. et al. Physical activity and fatigue in chronic obstructive pulmonary disease A population based study. Respir Med, v. 109, n. 8, p. 1048-57, Aug 2015. ISSN 1532-3064. Available at: < https://www.ncbi.nlm.nih.gov/pubmed/26070272 >.

BARREIRA, T. V. et al. Anthropometric correlates of total body fat, abdominal adiposity, and cardiovascular disease risk factors in a biracial sample of men and women. Mayo Clin Proc, v. 87, n. 5, p. 452-60, May 2012. ISSN 1942-5546. Available at: < https://www.ncbi.nlm.nih.gov/pubmed/22560524 >.

BELARDINELLI, R. et al. 10-year exercise training in chronic heart failure: a randomized controlled trial. J Am Coll Cardiol, v. 60, n. 16, p. 1521-8, Oct 2012. ISSN 1558-3597. Available at: < https://www.ncbi.nlm.nih.gov/pubmed/22999730 $>$.

BOUAZIZ, W. et al. Health benefits of endurance training alone or combined with diet for obese patients over 60: a review. Int J Clin Pract, v. 69, n. 10, p. 1032-49, Oct 2015. ISSN 17421241. Available at: < https://www.ncbi.nlm.nih.gov/pubmed/25963846 $>$.

BOWERS, M. T. Cardiovascular Health Among an Underserved Population: Clinical 
Perfil antropométrico e parâmetros cardiovasculares dos participantes de um programa de promoção da saúde de Araranguá/SC

Implications. Nurs Clin North Am, v. 50, n. 3, p. 457-64, Sep 2015. ISSN 1558-1357. Available at: $<$ https://www.ncbi.nlm.nih.gov/pubmed/26333603 $>$.

BRANT, L. C. et al. Relations of digital vascular function, cardiovascular risk factors, and arterial stiffness: the Brazilian Longitudinal Study of Adult Health (ELSA-Brasil) cohort study. J Am Heart Assoc, v. 3, n. 6, p. e001279, Dec 2014. ISSN 2047-9980. Available at: < https://www.ncbi.nlm.nih.gov/pubmed/25510401 >.

BRASIL. MINISTÉRIO DA SAÚDE. SECRETARIA DE VIGILÂNCIA EM SAÚDE. DEPARTAMENTO DE ANÁLISE DE SITUAÇÃO DE SAÚDE. Plano de ações estratégicas para o enfrentamento das doenças crônicas não transmissíveis (DCNT) no Brasil 2011-2022. Brasília: Ministério da Saúde: 160 p. 2011. Available at: < http://pesquisa.bvsalud.org/bvsms/resource/pt/mis-32487>

BRAWNER, C. A.; CHURILLA, J. R.; KETEYIAN, S. J. Prevalence of Physical Activity Is Lower among Individuals with Chronic Disease. Med Sci Sports Exerc, v. 48, n. 6, p. 1062-7, Jun 2016. ISSN 1530-0315. Available at: < https://www.ncbi.nlm.nih.gov/pubmed/26741117 >.

BURROWS, R. et al. High cardiometabolic risk in healthy Chilean adolescents: associations with anthropometric, biological and lifestyle factors. Public Health Nutr, v. 19, n. 3, p. 486-93, Feb 2016. ISSN 1475-2727. Available at: < https://www.ncbi.nlm.nih.gov/pubmed/25990645 >.

CAMPBELL, N. R.; NIEBYLSKI, M. L.; EXECUTIVE, W. H. L. Prevention and control of hypertension: developing a global agenda. Curr Opin Cardiol, v. 29, n. 4, p. 324-30, Jul 2014. ISSN 1531-7080. Available at: < https://www.ncbi.nlm.nih.gov/pubmed/25029450 >.

CHOW, C. K. et al. Prevalence, awareness, treatment, and control of hypertension in rural and urban communities in high-, middle-, and low-income countries. JAMA, v. 310, n. 9, p. 959-68, Sep 2013. ISSN 1538-3598. Available at: < https://www.ncbi.nlm.nih.gov/pubmed/24002282 >

DANIELE, T. M. et al. Associations among physical activity, comorbidities, depressive symptoms and health-related quality of life in type 2 diabetes. Arq Bras Endocrinol Metabol, v. 57, n. 1, p. 44-50, Feb 2013. ISSN 1677-9487. Available at: < https://www.ncbi.nlm.nih.gov/pubmed/23440098 $>$. 
Perfil antropométrico e parâmetros cardiovasculares dos participantes de um programa de promoção da saúde de Araranguá/SC

ECHOUfFO-TCHEUGUI, J. B. et al. Association of Physical Activity or Fitness With Incident Heart Failure: A Systematic Review and Meta-Analysis. Circ Heart Fail, v. 8, n. 5, p. 853-61, Sep 2015. ISSN 1941-3297. Available at: < https://www.ncbi.nlm.nih.gov/pubmed/26175539 >.

ENG, J. Y.; MOY, F. M.; BULGIBA, A. Impact of a Workplace Health Promotion Program on Employees' Blood Pressure in a Public University. PLoS One, v. 11, n. 2, p. e0148307, 2016. ISSN 1932-6203. Available at: < https://www.ncbi.nlm.nih.gov/pubmed/26840508 >.

GARCÍA-ORTIZ, L. et al. Relationship between objectively measured physical activity and cardiovascular aging in the general population--the EVIDENT trial. Atherosclerosis, v. 233, n. 2, p. 434-40, Apr 2014. ISSN 1879-1484. Available at: < https://www.ncbi.nlm.nih.gov/pubmed/24530775 $>$.

\section{GUIDELINES FOR DATA PROCESSING AND ANALYSIS OF THE INTERNATIONAL PHYSICAL ACTIVITY QUESTIONNAIRE -IPAQ, 2004. Available at: $<$ http://www.sdp.univ.fvg.it/sites/default/files/IPAQ English self-admin short.pdf $>$}

HARRIS, K. C. et al. Hypertension Canada's 2016 Canadian Hypertension Education Program Guidelines for Blood Pressure Measurement, Diagnosis, and Assessment of Risk of Pediatric Hypertension. Can J Cardiol, v. 32, n. 5, p. 589-97, May 2016. ISSN 1916-7075. Available at: < https://www.ncbi.nlm.nih.gov/pubmed/27118292 >.

HESSELINK, A. E. et al. A cluster-randomized controlled trial to study the effectiveness of a protocol-based lifestyle program to prevent type 2 diabetes in people with impaired fasting glucose. BMC Fam Pract, v. 14, p. 184, 2013. ISSN 1471-2296. Available at: < https://www.ncbi.nlm.nih.gov/pubmed/24295397 >.

JERÔNIMO, J. S. et al. [Physical activity in staff workers at Centers for Psychosocial Care in southern Brazil: temporal trends]. Cad Saude Publica, v. 30, n. 12, p. 2656-68, Dec 2014. ISSN 1678-4464. Available at: < https://www.ncbi.nlm.nih.gov/pubmed/26247994 >.

KNAPPER, J. T. et al. Time to Change Our Focus: Defining, Promoting, and Impacting Cardiovascular Population Health. J Am Coll Cardiol, v. 66, n. 8, p. 960-71, Aug 2015. ISSN 
Perfil antropométrico e parâmetros cardiovasculares dos participantes de um programa de promoção da saúde de Araranguá/SC

1558-3597. Available at: < https://www.ncbi.nlm.nih.gov/pubmed/26293767 >.

LEE, C. G. The emerging epidemic of hypertension in Asian children and adolescents. Curr Hypertens Rep, v. 16, n. 12, p. 495, Dec 2014. ISSN 1534-3111. Available at: < https://www.ncbi.nlm.nih.gov/pubmed/25304106 >.

LEE, I. M. et al. Effect of physical inactivity on major non-communicable diseases worldwide: an analysis of burden of disease and life expectancy. Lancet, v. 380, n. 9838, p. 219-29, Jul 2012. ISSN 1474-547X. Available at: < https://www.ncbi.nlm.nih.gov/pubmed/22818936 >.

LONG, G. H. et al. Temporal shifts in cardiovascular risk factor distribution. Am J Prev Med, v. 46, n. 2, p. 112-21, Feb 2014. ISSN 1873-2607. Available at: < https://www.ncbi.nlm.nih.gov/pubmed/24439344 >.

LOPRINZI, P. D.; DAVIS, R. E. Effects of individual, combined, and isolated physical activity behaviors on all-cause mortality and CVD-specific mortality: Prospective cohort study among U.S. adults. Physiol Behav, v. 151, p. 355-9, Nov 2015. ISSN 1873-507X. Available at: < https://www.ncbi.nlm.nih.gov/pubmed/26222613 >.

MAZALIN PROTULIPAC, J.; SONICKI, Z.; REINER, Ž. Cardiovascular disease (CVD) risk factors in older adults - Perception and reality. Arch Gerontol Geriatr, v. 61, n. 1, p. 88-92, 2015 Jul-Aug 2015. ISSN 1872-6976. Available at: < https://www.ncbi.nlm.nih.gov/pubmed/25944060 $>$.

MCLAUGHLIN, S. J.; CONNELL, C. M.; JANEVIC, M. R. Gender Differences in Trajectories of Physical Activity Among Older Americans With Diabetes. J Aging Health, v. 28, n. 3, p. 46080, Apr 2016. ISSN 1552-6887. Available at: < https://www.ncbi.nlm.nih.gov/pubmed/26187535 >.

MINDER, C. M. et al. Relation between self-reported physical activity level, fitness, and cardiometabolic risk. Am J Cardiol, v. 113, n. 4, p. 637-43, Feb 2014. ISSN 1879-1913. Available at: < https://www.ncbi.nlm.nih.gov/pubmed/24360775 $>$.

NACI, H.; IOANNIDIS, J. P. Comparative effectiveness of exercise and drug interventions on 
Perfil antropométrico e parâmetros cardiovasculares dos participantes de um programa de promoção da saúde de Araranguá/SC

mortality outcomes: metaepidemiological study. BMJ, v. 347, p. f5577, 2013. ISSN 1756-1833. Available at: < https://www.ncbi.nlm.nih.gov/pubmed/24473061 >.

NAKAMURA, P. M. et al. Health related quality of life is differently associated with leisure-time physical activity intensities according to gender: a cross-sectional approach. Health Qual Life Outcomes, v. 12, p. 98, 2014. ISSN 1477-7525. Available at: < https://www.ncbi.nlm.nih.gov/pubmed/25135321 >.

NORTHCOTT, J. M. et al. Adipokines and the cardiovascular system: mechanisms mediating health and disease. Can J Physiol Pharmacol, v. 90, n. 8, p. 1029-59, Aug 2012. ISSN 12057541. Available at: < https://www.ncbi.nlm.nih.gov/pubmed/22646022 >.

NUNES, A. P. et al. [Domains of physical activity and education in São Paulo, Brazil: a serial cross-sectional study in 2003 and 2008]. Cad Saude Publica, v. 31, n. 8, p. 1743-55, Aug 2015. ISSN 1678-4464. Available at: < https://www.ncbi.nlm.nih.gov/pubmed/26375652 >.

PATTYN, N. et al. The effect of exercise on the cardiovascular risk factors constituting the metabolic syndrome: a meta-analysis of controlled trials. Sports Med, v. 43, n. 2, p. 121-33, Feb 2013. ISSN 1179-2035. Available at: < https://www.ncbi.nlm.nih.gov/pubmed/23329606 >.

RASIAH, R. et al. The impact of physical activity on cumulative cardiovascular disease risk factors among Malaysian adults. BMC Public Health, v. 15, p. 1242, 2015. ISSN 1471-2458. Available at: < https://www.ncbi.nlm.nih.gov/pubmed/26673166 $>$.

RIBEIRO, A. G.; COTTA, R. M.; RIBEIRO, S. M. [The promotion of health and integrated prevention of risk factors for cardiovascular diseases]. Cien Saude Colet, v. 17, n. 1, p. 7-17, Jan 2012. ISSN 1678-4561. Available at: < https://www.ncbi.nlm.nih.gov/pubmed/22218533 >.

ROEVER, L. S. et al. Abdominal Obesity and Association With Atherosclerosis Risk Factors: The Uberlândia Heart Study. Medicine (Baltimore), v. 95, n. 11, p. e1357, Mar 2016. ISSN 1536-5964. Available at: < https://www.ncbi.nlm.nih.gov/pubmed/26986094 >.

SARMA, S. et al. The effect of physical activity on adult obesity: evidence from the Canadian NPHS panel. Econ Hum Biol, v. 14, p. 1-21, Jul 2014. ISSN 1873-6130. Available at: < 
Perfil antropométrico e parâmetros cardiovasculares dos participantes de um programa de promoção da saúde de Araranguá/SC

https://www.ncbi.nlm.nih.gov/pubmed/24958450 >.

SKRYPNIK, D. et al. Effects of Endurance and Endurance Strength Training on Body Composition and Physical Capacity in Women with Abdominal Obesity. Obes Facts, v. 8, n. 3, p. 2015. If5-87, ISSN 1662-4033. Available at: < https://www.ncbi.nlm.nih.gov/pubmed/25968470 $>$.

SOCIEDADE BRASILEIRA DE CARDIOLOGIA. $7^{\mathrm{a}}$ Diretriz Brasileira de Hipertensão Arterial. Arq. Bras de Cardiologia. vol. 107, nº. 3, supl. 3, 2016. Available at: < http://publicacoes.cardiol.br/2014/diretrizes/2016/05_HIPERTENSAO_ARTERIAL.asp>

SOCIEDADE BRASILEIRA DE CARDIOLOGIA. VI Diretrizes Brasileiras de Hipertensão Arterial. Arq. Bras. Cardiol. vol.95, supp.1, p.1-51, 2010. Available at: < http://publicacoes.cardiol.br/consenso/2010/Diretriz_hipertensao_associados.asp>

SUEMOTO, C. K. et al. Effects of body mass index, abdominal obesity, and type 2 diabetes on mortality in community-dwelling elderly in Sao Paulo, Brazil: analysis of prospective data from the SABE study. J Gerontol A Biol Sci Med Sci, v. 70, n. 4, p. 503-10, Apr 2015. ISSN 1758535X. Available at: < https://www.ncbi.nlm.nih.gov/pubmed/25209254 >.

THOMSON, J. L. et al. Participant adherence indicators predict changes in blood pressure, anthropometric measures, and self-reported physical activity in a lifestyle intervention: HUB city steps. Health Educ Behav, v. 42, n. 1, p. 84-91, Feb 2015. ISSN 1552-6127. Available at: < https://www.ncbi.nlm.nih.gov/pubmed/24986913 >.

TUCKER, C. M. et al. The effects of a culturally sensitive, empowerment-focused, communitybased health promotion program on health outcomes of adults with type 2 diabetes. J Health Care Poor Underserved, v. 25, n. 1, p. 292-307, Feb 2014. ISSN 1548-6869. Available at: < https://www.ncbi.nlm.nih.gov/pubmed/24509027 $>$.

VEDANTHAN, R. et al. Family-Based Approaches to Cardiovascular Health Promotion. J Am Coll Cardiol, v. 67, n. 14, p. 1725-37, Apr 2016. ISSN 1558-3597. Available at: < https://www.ncbi.nlm.nih.gov/pubmed/27056780 $>$. 
Perfil antropométrico e parâmetros cardiovasculares dos participantes de um programa de promoção da saúde de Araranguá/SC

VENTURELLI, M. et al. Effects of endurance, circuit, and relaxing training on cardiovascular risk factors in hypertensive elderly patients. Age (Dordr), v. 37, n. 5, p. 101, Oct 2015. ISSN 1574-4647. Available at: < https://www.ncbi.nlm.nih.gov/pubmed/26381921 >.

VÁZQUEZ, L. A. et al. Relationships between obesity, glycemic control, and cardiovascular risk factors: a pooled analysis of cross-sectional data from Spanish patients with type 2 diabetes in the preinsulin stage. BMC Cardiovasc Disord, v. 14, p. 153, 2014. ISSN 1471-2261. Available at: < https://www.ncbi.nlm.nih.gov/pubmed/25361574 >.

WANG, J. et al. The effectiveness of a community-based health promotion program for rural elders: a quasi-experimental design. Appl Nurs Res, v. 27, n. 3, p. 181-5, Aug 2014. ISSN $1532-$ 8201. Available at: < https://www.ncbi.nlm.nih.gov/pubmed/24440339 >.

WHO. HEALTH PROMOTION. 9TH GLOBAL CONFERENCE ON HEALTH PROMOTION SHANGHAI. Shanghai Declaration on promoting health in the 2030 Agenda for Sustainable Development 2016. Available at: < http://www.who.int/healthpromotion/conferences/9gchp/shanghai-declaration/en/ $>$ Acesso em: 08 maio 2017

WORLD HEALTH ORGANIZATION. Global recommendations on physical activity for health. WHO Library Cataloguing-in-Publication Data. World health organization; 2010. http://apps.who.int/bmi/index.jsp?introPage=intro 3.html. Acesso em: 08 maio 2017

WU, M. P. et al. Effectiveness of a community-based health promotion program targeting people with hypertension and high cholesterol. Nurs Health Sci, v. 14, n. 2, p. 173-81, Jun 2012. ISSN 1442-2018. Available at: < https://www.ncbi.nlm.nih.gov/pubmed/22380735 >.

ZHANG, Y.; PADMAN, R. Data-driven clinical and cost pathways for chronic care delivery. Am J Manag Care, v. 22, n. 12, p. 816-820, Dec 2016. ISSN 1936-2692. Available at: < https://www.ncbi.nlm.nih.gov/pubmed/27982668 $>$. 